\title{
Ultrapotent SARS-CoV-2 neutralizing antibodies effectively block new-emerging mutational variants
}

Aishun Jin ( $\nabla$ aishunjin@cqmu.edu.cn )

Chongqing Medical University

Tingting Li

Chongqing Medical University

Yingming Wang

Chongqing Medical University

Xiaojian Han

Chongqing Medical University

Chenjian Gu

Fudan University

Hangtian Guo

ShanghaiTech University

Kai Wang

Chongqing Medical University https://orcid.org/0000-0002-0137-1247

Fengjiang Liu

ShanghaiTech University

Feiyang Luo

Chongqing Medical University

Jie Hu

Chongqing Medical University

Chao Hu

Chongqing Medical University

Shenglong Li

Chongqing Medical University

Yanan Hao

Chongqing Medical University

Jingjing Huang

Chongqing Medical University

Yingyi Long

Chongqing Medical University

Shuyi Song

Chongqing Medical University

Wang Wang 
Chongqing Medical University

\section{Meiying Shen}

Harbin Medical University

\section{Ruixin Wu}

Chongqing Medical University

\section{Song $\mathrm{Mu}$}

Chongqing Medical University

\section{Qian Chen}

Chongqing Medical University

\section{Fengxia Gao}

Chongqing Medical University

Jianwei Wang

Chongqing Medical University

\section{Shunhua Long}

Chongqing Medical University

\section{Luo Li}

Chongqing Medical University

\section{Yang Wu}

Fudan University

\section{Yan Gao}

ShanghaiTech University

\section{Wei Xu}

Key Laboratory of Medical Molecular Virology (MOE/NHC/CAMS), School of Basic Medical Sciences, Fudan University, 130 Dong An Rd., Shanghai 200032

\section{Xia Cai}

Shanghai Medical College of Fudan University

\section{Di Qu}

Shanghai Medical College of Fudan University

\section{Qingzhu Gao}

Chongqing Medical University

\section{Guiji Zhang}

Chongqing Medical University

\section{Changlong $\mathrm{He}$}

Chongqing Medical University

\section{Wei Wang}

Chongqing Medical University

\section{Xiaoyun Ji}

Nanjing University

Ni Tang 
Chongqing Medical University

\section{Zhenghong Yuan}

Key Laboratory of Medical Molecular Virology, School of Basic Medical Sciences, Shanghai Medical

College, Fudan University https://orcid.org/0000-0003-0268-4891

\section{Youhua Xie}

Fudan University https://orcid.org/0000-0002-2416-7708

\section{Haitao Yang}

ShanghaiTech University

\section{Ai-Long Huang}

Chongqing Medical University https://orcid.org/0000-0003-0148-7423

\section{Article}

Keywords: SARS-CoV-2, mutations, variants

Posted Date: February 12th, 2021

DOl: https://doi.org/10.21203/rs.3.rs-215131/v1

License: (c) (i) This work is licensed under a Creative Commons Attribution 4.0 International License. Read Full License

Version of Record: A version of this preprint was published at Nature Communications on November 2nd, 2021. See the published version at https://doi.org/10.1038/s41467-021-26539-7. 


\section{Abstract}

Accumulating mutations on SARS-CoV-2 Spike (S) protein may increase the possibility of immune escape, challenging the present COVID-19 prophylaxis and clinical interventions. Here, in a panel of receptor binding domain (S-RBD) specific monoclonal antibodies (mAbs) with high neutralizing potency against authentic SARS-CoV-2, at least 6 of them were found to efficiently block the pseudovirus of 501Y.V2, a highly transmissible SARS-CoV-2 variant with escape mutations. The top 3 neutralizing Abs (13G9, 58G6 and 510A5) exhibited comparative ultrapotency as those being actively pursued for clinical development. Interestingly, the antigenic sites for the majority of our neutralizing Abs overlapped with a single epitope (13G9e) on S-RBD. Further, the 3-dimensional structures of 2 ultrapotent neutralizing Abs $13 \mathrm{G} 9$ or $58 \mathrm{G} 6$ in complex with SARS-CoV-2 S trimer demonstrated that both Abs bound to a steric region within $S^{472-490}$. Moreover, a specific linear region $\left(S^{450-457}\right)$ was identified as an additional target for 58G6. Importantly, our cryo-electron microscopy (cryo-EM) analysis revealed a unique phenomenon that the S-RBDs interacting with the fragments of antigen binding (Fabs) of 13G9 or $58 \mathrm{G} 6$ encoded by the IGHV1-58 and the IGKV3-20 gene segments were universally in the 'up' conformation in all observed particles. The potent neutralizing Abs presented in the current study may be promising candidates to fulfill the urgent needs for the current pandemic of SARS-CoV-2, and may of fundamental value for the next-generation vaccine development.

\section{Introduction}

The persistence of COVID-19 in the global population can result in the accumulation of specific mutations of SARS-CoV-2 with increased infectivity and/or enhanced possibilities of immune escape ${ }^{1-8}$. Two new SARS-CoV-2 variants, 501Y.V1 in the UK and 501Y.V2 in South Africa, exhibit high transmissibility 7,9 . Specific mutations of 501Y.V2 have been identified with reduced susceptibility to neutralization $3,4,6$. Continuous emergence of mutational variants as such has brought fundamental challenges to current clinical prophylaxis, and has raised great concerns of re-infection all over the world.

SARS-CoV-2 $S$ is a trimeric fusion protein responsible for viral entry ${ }^{10-12}$. As the core region for the host cell receptor ACE2 engagement, its S-RBD is the major target of clinical interventions ${ }^{11-13}$. Since the majority of SARS-CoV-2 S-RBD specific neutralizing Abs exert their protective functions by competing with ACE2, their epitopes are mainly located on the receptor binding motif (RBM, $S^{438-506}$ ) on S-RBD ${ }^{14,15}$. So far, most clinically available neutralizing Abs were directed towards the wild type SARS-CoV-2 S-RBD, which were dominant at the initial phase of COVID-19 pandemic ${ }^{16-26}$. However, the efficacy of these neutralizing Abs has been challenged by new-emerging mutational variants ever since. In particular, two mutations $\mathrm{S}^{\mathrm{K} 417 \mathrm{~N}}$ and $\mathrm{S}^{\mathrm{E} 484 \mathrm{k}}$ within 501Y.V2 S-RBD were recently found to be responsible for its escape from neutralization $3,4,6$. Such loss of sensitivity to immune response has caused the raising concerns about re-infection. Therefore, it becomes an urgent need to find effective mAbs that can neutralize SARSCoV-2 variants with escape mutations as 501Y.V2, for the control of current COVID-19 prevalence. 
Recent cryo-EM analysis has demonstrated that each $S$ protein trimer can form a natural conformation with the mixture of 3 S-RBDs in the 'down' state or the 1 'up' and 2 'down' state ${ }^{11}$. Thus far, majority of reported S-RBD specific neutralizing Abs were found to form complexes with S-RBDs either as the natural conformation of the $S$ trimer, or in a mixture of the 2 'up' ( 1 'down') state and the 3 'up' state ${ }^{17-21,26-28}$. However, the correlation between the various states of S-RBDs in the complex and the potency of corresponding Abs in blocking SARS-CoV-2 remains unknown.

Here, we focused on 20 neutralizing Abs selected from a SARS-CoV-2 S-RBD specific mAb reservoir and confirmed their potency against the authentic virus. Excitingly, at least 6 of our neutralizing Abs showed marked efficacy against the 501Y.V1 and 501Y.V2 pseudoviruses. In addition, we found that 13G9e on SRBD was accounted for 16 out of these 20 neutralizing Abs against SRAS-CoV-2. While 58G6 was found to target a specific linear region $\left(S^{450-457}\right)$, detailed cryo-EM analysis demonstrated that $13 \mathrm{G} 9$ and $58 \mathrm{G} 6$ both recognized steric sites within $S^{472-490}$ on S-RBD. Uniquely, their Fabs were shown to interact with all 3 S-RBDs in the 'up' state in all Fab-S trimer complexes observed in our study. Our study presents a panel of neutralizing $A b$ candidates for the current clinical development of effective therapeutic strategies. And the identified novel neutralizing mechanism might provide updated information for the vaccine design against the present COVID-19 pandemic.

\section{Results}

\section{Sustained efficacy of SARS-CoV-2 S-RBD specific neutralizing Abs in blocking 501Y.V2 pseudovirus}

By our recently established rapid neutralizing Abs screening system ${ }^{29}$, we have successfully obtained 20 neutralizing Abs with high affinities to S-RBD from COVID-19 convalescent plasma. Here, the neutralizing potencies of these 20 antibodies were confirmed by the half inhibition concentrations $\left(\mathrm{IC}_{50}\right)$ against authentic SARS-CoV-2 quantified via RT-qPCR. As our most potent mAb, $13 \mathrm{G} 9$ exhibited striking neutralizing efficacy, with the $\mathrm{IC}_{50}$ value of $0.007 \mu \mathrm{g} / \mathrm{mL}$ (Fig. 1a and Extended Data Table. 1). Similar ultrapotency was shown for 2 other top neutralizing Abs, 58G6 and 510A5, which demonstrated identical $\mathrm{IC}_{50}$ values as our previous findings ${ }^{29}$ (Fig. 1a and Extended Data Table. 1). The other $17 \mathrm{mAbs}$ could also efficiently neutralize authentic SARS-CoV-2, all displaying acceptable potency for practical development (Fig. 1a, Extended Data Fig. 1 and Extended Data Table. 1).

Given the high transmissibility and reduced neutralization susceptibility associated with SARS-CoV-2 variants, we tested the impact of newly emerged S-RBD mutations to the top $10 \mathrm{mAbs}$. For this purpose, we obtained pseudoviruses carrying the S protein of the wild type SARS-CoV-2 or 501Y.V1, together with pseudovirus bearing a chimeric construct including the S-RBD mutations associated with 501Y.V2 $\left(S^{K 417 N}, S^{E 484 K}\right.$ and $S^{N 501 Y}$ ) in addition to the dominant mutation $S^{D 614 G}$ of the $S$ protein. First, the efficacy of all 10 mAbs against the wild type pseudovirus was confirmed (Fig. 1b and Extended Data Table. 1). And we verified that these neutralizing Abs could directly inhibit the interaction of S-RBD and ACE2, by competitive ELISA and surface plasmon resonance (SPR) assay (Extended Data Figs. 2 and 3). 
Further, we found that all of these 10 mAbs retained their neutralizing potency against the 501Y.V1 pseudovirus (Fig. 1b). Importantly, the S-RBD escape mutations of 501Y.V2 did not compromise the efficacy of 5 of our potent neutralizing Abs (58G6, 510A5, 510A4, 51D3 and 55A8), while it only slightly increased the $\mathrm{IC}_{50}$ value of $13 \mathrm{G} 9$ to $0.033 \mu \mathrm{g} / \mathrm{mL}$ (Fig. 1b). Two other mAbs, $51 \mathrm{D} 7$ and 81E1, also exhibited neutralization for 501Y.V2 pseudovirus, though both showed apparent decrease in their activities (Fig. 1b). We found that the neutralizing capabilities of 51E10 and 52F7 were impaired by 501Y.V2 pseudovirus, which was similar to a wide range of reported S-RBD specific neutralizing Abs $3,4,6$ (Fig. 1b). Collectively, we obtained 20 neutralizing Abs with high potency against authentic SARS-CoV-2, and at least 6 of them retained their efficacy against 501Y.V2 pseudovirus. These results indicate that our neutralizing Abs may be promising candidates for the interventions against the constant evolving SARSCoV-2.

\section{The identification of epitopes on S-RBD for selected neutralizing Abs}

To elucidate potential mechanisms of our neutralizing Abs against SARS-CoV-2, we investigated the antigenic landscape of S-RBD with the above 20 neutralizing Abs with additional 54 mAbs from our developed S-RBD-specific mAb reservoir, by competitive ELISA. As shown in Fig. 2a, 5 clusters of mAbs were identified according to their recognition sites, with the mAbs from each cluster competed for 13G9e, the epitope recognized by a non-neutralizing SARS-CoV-2 specific mAb 81A11 (81A11e) or by a SARSCoV specific neutralizing Ab CR3022 (CR3022e) (Fig. 2a). Interestingly, the epitopes recognized by the majority of all mAbs with confirmed neutralizing potency ${ }^{29}$ overlapped with $13 \mathrm{G} 9 \mathrm{e}$, including 16 of the above 20 mAbs (Fig. 2a). To further assess the interrelationships between the 20 neutralizing Abs in detail, we biotinylated them and performed competitive ELISA. We found that $80 \%$ of these mAbs competed with 13G9, whereas the antigenic sites of the other 4 neutralizing Abs (510A5, 55A8, 57F7 and 07C1) overlapped with an independent epitope (510A5e) (Extended Data Fig. 4). These findings suggest that there may be at least two different epitopes on S-RBD responsible for SARS-CoV-2 neutralization, from which $13 \mathrm{G} 9$ e might represent a key antigenic site for the binding of neutralizing Abs to S-RBD.

To test whether our mAbs could elicit synergistic effect against authentic SARS-CoV-2, we paired each of the top 3 neutralizing Abs (13G9, 58G6 and 510A5) with individual mAbs from different clusters, as shown in Fig. 2a. We observed synergistic effects with different combinations of all 3 neutralizing Abs for complete neutralization, confirming the advantages of neutralizing Ab cocktails (Fig. 2b-d). Of note, adding neutralizing $A b$ from a different cluster barely reduced the $\mathrm{IC}_{50}$ of the top $3 \mathrm{mAbs}$, indicating that our potent mAbs alone were sufficient in neutralizing SARS-CoV-2 (Fig. 2b-d).

\section{The unique cryo-EM structure of the $13 \mathrm{G} 9$ or the $58 \mathrm{G} 6$ Fab-S trimer complex}

To identify the precise antigenic sites for the potent neutralizing Abs, we analyzed the cryo-EM structures

of the Fabs derived from $13 \mathrm{G} 9$ or $58 \mathrm{G} 6$ in complex with the modified $S$ trimer with stabilizing mutations ${ }^{12}$ (Extended Data Fig. 5a, b). The high-quality electron density maps were generated via single-particle analysis of the $13 \mathrm{G} 9$ Fab-S trimer complex, the overall resolution of which was finally refined to $3.9 \AA$ 
(Fig. 3a, Extended Data Fig. 5c-e and Extended Data Table. 2). Notably, of all observed Fab-S trimer complex, only one conformation of S trimer with 3 'up' S-RBDs was found, complexed with 3 Fabs (Fig. 3a and Extended Data Table. 2). Although the electron density for the 13G9 Fab was visible, it was blurred as a result of molecular flexibility, thus the Fab model was predicted using Phyre $2^{30}$ and manually fitted into the cryo-EM map. The structural analysis revealed that the S-RBD loop $\left(S^{472-490}\right)$ was accounted for the binding of 13G9 (Fig. 3b). Docking the 13G9 crystal structure in the cryo-EM map indicated that the complementarity determining region of the light chain (CDRL), as well as the complementarity determining region 1 and 3 of the heavy chain ( $\mathrm{CDRH} 1$ and $\mathrm{CDRH} 3$ ) all contacted with $\mathrm{S}^{472-490}$ (Fig. 3c).

Next, we solved the cryo-EM structure of $58 \mathrm{G} 6$ Fabs in complex with the S trimer, at $3.6 \AA$ resolution (Fig. 4a, Extended Data Fig. 5f-h and Extended Data Table. 2). Interestingly, the structure of such complex was found to be similar to that of 13G9, with only one dominant particle class corresponding to a 3-Fabbound complex with all S-RBDs in the 'up' conformation (Fig. 4a). Likewise, each $58 \mathrm{G} 6$ Fab targeted an SRBD in a very similar way to that of $13 \mathrm{G} 9 \mathrm{Fab}$ (Fig. $4 \mathrm{a}$ ). $58 \mathrm{G} 6$ also used its CDRL, together with CDRH1 and CDRH3 to recognize $S^{472-490}$ of SARS-CoV-2, where all of them contributed to the paratope buried surface area (Fig. 4b, c).

In order to explain such similarity between $13 G 9$ and 58G6, we analyzed the gene clusters encoding these 2 mAbs. We found that both 13G9 and 58G6 were encoded by IGHV1-58 for the heavy chain and by IGKV3-20 for the light chain, with a few differences in amino acids of their CDRH3 and CDRL3 (Extended Data Table. 3). Notably, several steric differences were observed in $\mathrm{CDRH}$ of the two neutralizing Abs, which were largely determined by $\mathrm{CDRH} 2$ and $\mathrm{CDRH} 3$ (Fig. 3c, 4c). Such differences might be accounted for the distinguished steric binding site on S-RBD corresponding to 13G9 and 58G6. As shown in Fig. 3d and 4d, a potential steric clash between 13G9 Fab or 58G6 Fab and ACE2 was observed, indicating a possible neutralizing mechanism of these 2 neutralizing Abs. Taken together, these Fab-S complex structures suggest that our Abs 13G9 and 58G6 adopt the same potential neutralizing mechanism, wherein they are more competent to bind to S-RBDs in the all 'up' state, occluding the access of SARSCoV-2 S to ACE2.

\section{A linear binding region on S-RBD recognized by $58 \mathrm{G} 6$}

Independently, precise interactive regions of our potent neutralizing Abs were determined by profiling their linear binding sites on SARS-CoV-2 S-RBD. We assessed the binding capability of the 20 mAbs to the denatured S-RBD via Western Blot analysis. In the preliminary screen, $9 \mathrm{mAbs}$ were found to bind to the denatured S-RBD (Extended Data Fig. 6). Next, we designed and synthesized fifteen 20-mer peptides (RBD1 to RBD15), overlapping with 5 amino acids, to cover the entire S-RBD ( S $^{319-541}$ ) (Extended Data Fig. 7a). Unexpectedly, we found that the simultaneous recognition of RBD2, RBD9 and RBD13 were shared by 5 of these 9 mAbs binding to denatured S-RBD, while $58 \mathrm{G} 6$ only bound to RBD9 $\left(\mathrm{S}^{439-459}\right)$ (Extended Data Fig. 7b, c). To determine the essential amino acid residues in S-RBD accounted for 58G6 binding, we re-synthesized two 20-mer peptides overlapping with 15 amino acids (RBD9-1 and RBD9-2), 
covering the RBD9 specific residues (Extended Data Fig. 7a). The results of peptide ELISA showed that 58G6 formed a stronger interaction with RBD9-1 than RBD9, in a dose-dependent manner (Fig. 5a, b). When we individually replaced each amino acid residue in RBD9-1 ( $\left.S^{444-463}\right)$ with alanine $(A)$, we found that the binding of $58 \mathrm{G} 6$ to 7 amino acid residues $\left(\mathrm{S}^{450-457}\right)$ was significantly reduced (Fig. 5a). To a lesser extent, $S^{445-449}$ also affected the binding activity of $58 \mathrm{G} 6$, which might explain for the abolished interaction of 58G6 to RBD9-2 (Fig. 5a). Hence, $S^{450-457}$ represented an essential region of S-RBD for the recognition of neutralizing Abs. Moreover, we found that RBD9-1 bound to ACE2 dose-dependently, which could be competitively inhibited by $58 \mathrm{G} 6$ (Fig. $5 \mathrm{c}-\mathrm{e}$ ). And the region of $\mathrm{S}^{445-463}$ was identified to be critical for the RBD9-1-ACE2 interaction (Fig. 5c, d). It is worth mentioning that the interaction of 13G9 or 510A5 to the denatured S-RBD was not observed (Extended Data Fig. 6). Taken together, we evidenced a second region on S-RBD $\left(S^{450-457}\right)$, which could be recognized by $58 \mathrm{G} 6$ in the linearized form.

\section{Discussion}

The pandemic of COVID-19 has led to immunological adaptation of SARS-CoV-2 and generation of mutational variants ${ }^{3,4,6-8}$. Newly emerged 501Y.V2 in South Africa has been reported to confer resistance to neutralization from multiple available mAbs, convalescent plasma and vaccinee sera, posting a high re-infection risk ${ }^{3,4,6}$. In the present study, we identified 20 neutralizing Abs with high potency against the authentic virus, from a SARS-CoV-2 S-RBD specific mAb reservoir. Among them, 13G9, 58G6 and 510A5 all exhibited ultrapotency against SARS-CoV-2, comparable to most effective neutralizing Abs reported up to date ${ }^{16-23,26}$. Remarkably, at least 6 of our neutralizing Abs demonstrated satisfying efficacy against the $501 \mathrm{Y} . \mathrm{V} 2$ pseudovirus, including the 3 ultrapotent mAbs. These findings highlighted their potential in the prevention and treatment for the COVID-19 pandemic, particularly for the current SARS-CoV-2 mutational variants.

In order to understand the sustained potency of our neutralizing Abs against S-RBD mutations, we thoroughly analyzed their antigenic sites. Interestingly, we found that the epitopes recognized by the majority of our S-RBD specific mAbs overlapped with 13G9e, suggesting that it represented one of the vulnerable sites on SARS-CoV-2 S-RBD. Further, the cryo-EM analysis demonstrated a key region of $\mathrm{S}^{472-}$ 490 on S-RBD, recognized by the most potent neutralizing Ab 13G9. And we directly evidenced that such steric region could also be recognized by the ultrapotent $58 \mathrm{G} 6$, with the significance of this region independently demonstrated by another group ${ }^{26}$. Considering $S^{472-490}$ takes part in the composition of the ACE2 recognition site on S-RBD ${ }^{10,13,31}$, it is not surprise to find 2 of our ultrapotent neutralizing Abs targeting this region. Despite the fact that 501Y.V2 carries a mutation within $S^{472-490}\left(S^{E 484 K}\right)$ correlated with the reduced sensitivity to neutralization, it does not seem to compromise the efficacy of our ultrapotent neutralizing Abs. We noted that 13G9 and 58G6 were both transcribed from IGHV1-58 and IGKV1-20, which were also genetically responsible for another reported neutralizing Ab with high potency ${ }^{26}$. These findings highlighted the otherwise overlooked importance of IGHV1-58 and IGKV1-20 
germlines restricted Abs against SARS-CoV-2 and 501Y.V2, as they could form a class with highly similar epitopes represented by $S^{472-490}$.

To our surprise, a parallel linear epitope screening analysis revealed that $58 \mathrm{G} 6$ also recognized a linear region on denatured S-RBD with amino acid residues of $S^{450-457}$, in addition to the steric region within $S^{472-490}$ identified above. Given the significance of this linear region for the interaction of $58 \mathrm{G} 6$ to S-RBD in the denatured form, we speculated that the amino acid residues of $S^{450-457}$ might also exhibit relative importance for $58 \mathrm{G} 6$ in the natural form. Of note, recent cryo-EM structure analysis has revealed 3 key ACE2-interacting amino acid residues $\left(S^{\mathrm{Y} 453}, S^{L 455}\right.$, and $\left.S^{\mathrm{F} 456}\right)$ within this linear region ${ }^{10,13,31}$, indicating $S^{450-457}$ may be another critical site for potent S-RBD specific neutralizing Ab. Together with $S^{472-490}$, these two regions may constitute the precise antigenic site for the ultrapotent neutralization of $58 \mathrm{G} 6$ against SARS-CoV-2 and its mutational variants. Although certain steric proximity of $13 G 9$ to $S^{450-457}$ was observed, it needed to be pointed out that no specific linear binding sites were identified for this mAbs. Such findings suggested that the specific inclusion of $S^{450-457}$ in the epitope recognized by $58 \mathrm{G} 6$ might be correlated to its marked advancement of neutralizing potency against 501Y.V2 relative to $13 \mathrm{G} 9$.

Upon the identification of SARS-CoV-2 S trimer structure via cryo-EM analysis, the presence of S-RBD 'down' conformation has been found in the Fab-S trimer complexes of most, if not all, reported SARS-CoV2 neutralizing $A \mathrm{Abs}^{17-23,26,27}$. For the ultrapotent $13 \mathrm{G} 9$ or $58 \mathrm{G} 6$, one unique finding in our study is that the S-RBDs interacting with the Fabs of these Abs are universally in the 'up' state, in all observed particles. We speculate that the conformational shift of S-RBD from the 'down' to the 'up' state may result from the steric fitness of $13 \mathrm{G} 9$ or $58 \mathrm{G} 6$ to the corresponding epitope within each S-RBD. And such conformation in each complex could render S-RBD completely inaccessible for ACE2. This mechanism might, to a certain extent, explain the ultrapotency of 13G9 or $58 \mathrm{G} 6$ in neutralizing SARS-CoV-2 and its mutational variants with enhanced infectivity and/or reduced sensitivity to neutralization.

In conclusion, we present a panel of potent SARS-CoV-2 S-RBD specific neutralizing Abs with satisfying efficiency against 501Y.V2, for which a significant proportion of reported neutralizing Abs are impaired. We demonstrate that neutralizing Abs derived from the plasma of the convalescent patient can promote the conformational shift of all 3 S-RBDs to the 'up' state, probably leading to the occlusion of ACE2 access. The specific conformation revealed in our study may represent an eminent mechanism of neutralization for SARS-CoV-2 S-RBD specific mAbs. Our broad-spectrum neutralizing Abs and mAbs functioning through similar mechanisms to that of $13 \mathrm{G} 9$ or $58 \mathrm{G} 6$ may be promising candidates for the prophylaxis and therapeutic interventions of the pandemic of SARS-CoV-2 variants carrying escape mutations. Our findings may provide vital information to incorporate into the next-generation vaccine development.

\section{Materials And Methods}

\section{Antibodies}


The human antibodies studied in this paper were isolated from 39 SARS-CoV-2 convalescent patient plasma in China and the original clinical studies to obtain blood samples after written informed consent were previously described ${ }^{29}$ and had been approved by the Ethics Board of ChongQing Medical University. The antibodies were isolated using flow sorting for isolation and cloning of single antigenspecific $B$ cells and the antibody variable genes encoding monoclonal antibodies ${ }^{29}$.

\section{Recombinant antibody production and purification}

A pair of plasmids separately expressing the heavy- and light- chain of antibodies were transiently cotransfected into Expi293 ${ }^{\text {TM }}$ cells (Catalog No. A14528, ThermoFisher) with ExpiFectamine ${ }^{\text {TM }} 293$ Reagent. Then the cells were cultured in shaker incubator at $120 \mathrm{rpm}$ and $8 \% \mathrm{CO}_{2}$ at $37^{\circ} \mathrm{C}$. After 7 days, the supernatants with the secretion of antibodies were collected and captured by protein G Sepharose (GE Healthcare). The bound antibodies on the Sepharose were eluted and dialyzed into phosphate-buffered saline (PBS). The purified antibodies were used in following binding and neutralization analyses.

\section{Sequence analysis of antigen-specific mAb}

IMGT/V-QUEST (http://www.imgt.org/ IMGT_vquest /vquest) and IgBLAST (https://www.ncbi.nlm.nih.gov/igblast/), MIXCR (https://mixcr.r eadthedocs.io/en/master/) and VDJtools (https://vdjtools-doc.readthedocs.io/en/master/overlap.html) tools were used to do the variable region analysis and annotation for each antibody clone.

\section{The competition with ACE2 measured by SPR}

To determine the competition with the human ACE2 peptidase domain, SARS-CoV-2 S-RBD was coated on a CM5 sensor chip via amine group for a final RU around 250 . Antibodies $(20 \mu \mathrm{g} / \mathrm{mL})$ were injected onto the chip until binding steady-state was reached. ACE2 $(20 \mu \mathrm{g} / \mathrm{mL})$ was then injected for 60 seconds. Blocking efficacy was determined by comparison of response units with and without prior antibody incubation.

\section{Competitive ELISA}

For the competitive ELISA used in epitope mapping of $m A b s, 2 \mu \mathrm{g} / \mathrm{mL}$ recombinant S-RBD-his (Sino Biological, Beijing, China) was added in 384-well plates and incubated at $4{ }^{\circ} \mathrm{C}$ overnight. $50 \mu \mathrm{g} / \mathrm{mL} \mathrm{mAbs}$ per well were added. The plates were incubated at $37^{\circ} \mathrm{C}$ for $1 \mathrm{~h}$ and then washed. Biotinylation of mAbs (the 20 neutralizing Abs and 81A11, previously reported SARS-CoV CR3022 ${ }^{32}$ were performed using the EZ-link NHS-PEO Solid Phase Biotinylation Kit (Pierce) according to the manufacturer's protocol and purified using MINI Dialysis Unit (ThermoFisher, 69576). $500 \mathrm{ng} / \mathrm{mL}$ biotinylated mAbs were added to each well, and the plates were incubated at $37^{\circ} \mathrm{C}$ for $1 \mathrm{~h}$. ALP-conjugated Streptavidin (Mabtech, Sweden, 3310-10) was added at 1:1000, followed by an incubation of $30 \mathrm{~min}$ at $37^{\circ} \mathrm{C}$. Quantification step was the same as described above.

\section{Peptide ELISA}


Peptide ELISA was performed with synthesized peptides overlapping with 5 amino acids (Genescripts, Wuhan, China). These peptides were tethered by N-terminal biotinylated linker peptides (biotin-ahx), except for the first peptide at the $\mathrm{N}$-terminus, whose biotin was linked to the $\mathrm{C}$ terminus instead. The RBD9-1 amino acid residues were selected and mutated to alanine and synthesized by Genescripts (Wuhan, China). $50 \mu \mathrm{L}$ synthesized peptide was added to the streptavidin-coated 384-well plate in duplets to make a final concentration of $5 \mu \mathrm{g} / \mathrm{mL}$. The plates were incubated for $2 \mathrm{hrs}$ at RT. After washing, the plates were blocked with Protein-Free Blocking Buffer (Pierce, USA, 37573) at RT for $1 \mathrm{~h}$ and incubated with $10 \mu \mathrm{g} / \mathrm{mL}$ testing mAbs at RT for another $1 \mathrm{~h}$. Reacted mAbs were detected using ALP-conjugated Goat F(ab')2 Anti-Human (IgG (Fab')2) secondary antibody (Abcam, ab98532, 1:2000) for 30 min at RT, followed with quantification detection.

For the ACE2 competitive peptide ELISA, $5 \mu \mathrm{g} / \mathrm{mL}$ synthesized RBD9-1 was immobilized on the streptavidin-coated 384-well plate at RT for $2 \mathrm{hrs}$. After washing with Protein-Free Blocking Buffer, the plates were blocked with this blocking buffer. Next, serial diluted $58 \mathrm{G} 6(20-0.625 \mu \mathrm{g} / \mathrm{mL})$ in $50 \mu \mathrm{L}$ of the blocking buffer were added into plate and the plates were incubated at RT for $1 \mathrm{~h}$. Then, the plate incubated with $2 \mu \mathrm{g} / \mathrm{mL}$ ACE2 at RT for another $1 \mathrm{~h}$. The ELISA plates were washed 4 times by blocking buffer and $50 \mu \mathrm{L}$ Goat $F\left(a^{\prime}\right) 2$ Anti-Human ( $\mathrm{IgG}\left(\mathrm{Fab}^{\prime}\right) 2$ ) secondary antibody conjugated with ALP (Abcam, ab98532, 1:2000) was incubated with the plate at RT for 30 min. The plate was washed and followed with quantification detection.

\section{Production of S protein pseudovirus}

pVSVG expressing SARS-CoV-2 spike was constructed as previously described ${ }^{33}$. The packaging plasmid (VSV-G pseudotyped $\triangle$ G-luciferase) encoding either SARS-CoV-2 S, 501Y.V1 S and chimeric construct including 501Y.V2 S-RBD and $S^{D 614 G}$ was generated. HEK293T cells were grown to $80 \%$ confluency before transfection with VSV-G pseudotyped $\triangle G$-luciferase, pWPXL and PSPAX2. Cells were cultured overnight at $37^{\circ} \mathrm{C}$ with $5 \% \mathrm{CO}_{2}$. DMEM supplemented with $5 \%$ fetal bovine serum and $100 \mathrm{I} . \mathrm{U} . / \mathrm{mL}$ of penicillin and $100 \mu \mathrm{g} / \mathrm{mL}$ of streptomycin were added to the inoculated cells, which were cultured overnight for $72 \mathrm{hrs}$. The supernatant was harvested, filtered by $0.45 \mu \mathrm{m}$ filter and centrifugated at $300 \mathrm{~g}$ for 10 mins to collect the supernatant and then aliquoted and storied at $-80^{\circ} \mathrm{C}$.

\section{Pseudovirus neutralization assay}

$50 \mu \mathrm{L}$ serially diluted mAbs were incubated with the same volume of the HEK293T cell supernatants containing pseudovirus for $1 \mathrm{~h}$ at $37^{\circ} \mathrm{C}$. These pseudovirus-antibody mixtures were added to ACE2 expressing HEK293T cells (HEK293T/ACE2). After $72 \mathrm{hrs,} \mathrm{the} \mathrm{luciferase} \mathrm{activities} \mathrm{of} \mathrm{infected}$ HEK293T/ACE2 cells were detected by the Bright-Luciferase Reporter Assay System (Promega, E2650). The $\mathrm{IC}_{50}$ of the evaluated mAbs were tested by the Varioskan LUX Microplate Spectrophotometer (Thermo Fisher), and calculated by a four-parameter logistic regression using GraphPad Prism 8.0.

\section{Authentic SARS-CoV-2 neutralization assay}


This assay was performed in a biosafety level 3 laboratory of Fudan University. Serially diluted mAbs were incubated with authentic SARS-CoV-2 (nCoV-SH01, GenBank: MT121215.1, 100 TCID50) for $1 \mathrm{~h}$ at $37^{\circ} \mathrm{C}$. After the incubation, the mixtures were then transferred into 96-well plates, which were seeded with Vero E6 cells. The plates were kept at $37^{\circ} \mathrm{C}$ for $48 \mathrm{hrs}$ and each well was visually assessed for CPE. And the supernatant viral RNA load of each well was quantified by RT-qPCR.

For RT-qPCR, the viral RNA was extracted from the collected supernatant using Trizol LS (Invitrogen) and used as templates for the RT-qPCR analysis by Verso 1-Step RT-qPCR Kit (Thermo Scientific) following the manufacturer's instructions. PCR primers targeting SARS-CoV-2 N gene (nt 608-706) were as followed, forward: 5'-GGGGAACTTCTCCTGCTAGAAT-3', and reverse: 5'-CAGACATTTTGCTCTCAAGCTG-3'. RT-qPCR was performed using the LightCycler 480 II PCR System (Roche) with the following program: $50^{\circ} \mathrm{C} 15$ $\min ; 95^{\circ} \mathrm{C} 15 \mathrm{~min} ; 40 \mathrm{cycles}$ of $95^{\circ} \mathrm{C} 15 \mathrm{sec}, 50^{\circ} \mathrm{C} 30 \mathrm{sec}, 72^{\circ} \mathrm{C} 30 \mathrm{sec}$.

\section{Western blot analysis}

The recombinant S-RBD protein was mixed with $5 \times$ loading buffer (Beyotime, Shanghai, China) and denatured for $5 \mathrm{~min}$ at $100^{\circ} \mathrm{C}$. Denatured proteins ( $200 \mathrm{ng}$ ) were subjected to electrophoresis with $10 \%$ SDS-polyacrylamide gel and then transferred to PVDF membranes. After blocking by skim milk (Biofroxx), the membranes were incubated at $4{ }^{\circ} \mathrm{C}$ overnight, with the purified mAbs as primary Abs. The next days, the membranes were washed with TBST and incubated with HRP-conjugated Goat-anti-human Fc antibody (Abcam, ab99759, 1:10000) for $1 \mathrm{~h}$ at RT. The membranes were examined on Bio-rad ChemiDoc Imaging System (Bio-rad).

\section{Protein expression and purification}

To express the prefusion spike ectodomain, the gene encoding residues 1-1208 of SARS-CoV-2 spike (GenBank: MN908947.3) with a C-terminal T4 fibritin trimerization motif, an HRV-3C protease cleavage site, a Twin-Strep-tag and an 8xHis-tag was synthesized, and cloned into the mammalian expression vector pcDNA3.1, which was a kind gift from L. Sun at Fudan University, China. The gene of spike protein was constructed with proline substitutions at residues 986 and 987, a "GSAS" instead of "RRAR" at the furin cleavage site (residues 682-685) according to Jason S. McLellan's research ${ }^{12}$.

Expi293 cells (Thermo Fisher Scientific, USA) cultured in Freestyle 293 Expression Medium (Thermo Fisher Scientific, USA) were maintained at $37^{\circ} \mathrm{C}$. Cells were diluted to a density of $2.5 \times 10^{6}$ to $3 \times 10^{6}$ cells per $\mathrm{ml}$ before transfection. For protein production, $1.2 \mathrm{mg}$ DNA was mixed with $3 \mathrm{mg}$ polyethyleneimine in $30 \mathrm{~mL}$ Freestyle 293 Expression Medium, incubated for $20 \mathrm{~min}$, then added to 1000 $\mathrm{mL}$ of cells ${ }^{34}$. Transfected cells were cultured at $35^{\circ} \mathrm{C}$, and the cell culture supernatant was collected at day 4 to day 5 .

Protein was purified from filtered cell supernatants using Strep-Tactin resin (IBA) before being subjected to additional purification by gel filtration chromatography using a Superose $610 / 300$ column (GE Healthcare, USA) in 1×PBS, pH 7.4 (Extended Data Fig. 5a, b). 


\section{Cryo-EM sample preparation and data collection}

Purified SARS-CoV-2 spike was diluted to a concentration of $1.5 \mathrm{mg} / \mathrm{mL}$ in PBS, pH 7.4. $5 \mu \mathrm{L}$ of purified SARS-CoV-2 S was mixed with $1 \mu \mathrm{L}$ of $58 \mathrm{G} 6 \mathrm{Fab}$ fragments at $2 \mathrm{mg} / \mathrm{ml}$ in PBS and incubated for $30 \mathrm{~min}$ on ice. A $3 \mu \mathrm{L}$ aliquot of the mixture (added with $0.01 \%$ DDM) was applied onto an $\mathrm{H}_{2} / \mathrm{O}_{2}$ glowdischarged, 300-mesh Quantifoil R1.2/1.3 grid (Quantifoil, Micro Tools GmbH, Germany). The grid was then blotted for $3.0 \mathrm{~s}$ with a blot force of -1 at $8{ }^{\circ} \mathrm{C}$ and $100 \%$ humidity and plunge-frozen in liquid ethane using a Vitrobot (Thermo Fisher Scientific, USA). Cryo-EM data sets were collected at a $300 \mathrm{kV}$ Titan Krios microscope (Thermo Fisher Scientific, USA) equipped with a K3 detector (Gatan, USA). The exposure time was set to $2.4 \mathrm{~s}$ with a total accumulated dose of 60 electrons per $\AA^{2}$, which yields a final pixel size of $0.82 \AA .2605$ micrographs were collected in a single session with a defocus range comprised between 1.0 and $2.8 \mu \mathrm{m}$ using SerialEM. The sample preparation and data collection for the SARS-CoV-2 S-13G9 Fab complex were in accordance with the SARS-CoV-2 S-58G6 Fab complex. The statistics of cryo-EM data collection can be found in Extended Data Table 2.

\section{Cryo-EM data processing}

All dose-fractioned images were motion-corrected and dose-weighted by MotionCorr2 software ${ }^{35}$ and their contrast transfer functions were estimated by cryoSPARC patch CTF estimation ${ }^{36}$. For the dataset of SARS-CoV-2 S-58G6 Fab complex, a total of 1,255,599 particles were auto-picked using the template picker and 820,872 raw particles were extracted with a box size of 512 pixels in cryoSPARC ${ }^{36}$. The following 2D, 3D classifications, and refinements were all performed in cryoSPARC. 237,062 particles were selected after two rounds of 2D classification, and these particles were used to do Ab-Initio reconstruction in six classes. Then these six classes were used as 3D volume templates for heterogeneous refinement with all selected particles, with 108,020 particles converged into the SARS-CoV2 S-58G6 Fab class. Next, this particle set was used to perform non-uniform refinement, yielding a resolution of $3.56 \AA$.

For the dataset of SARS-CoV-2 S-13G9 Fab complex, a total of 445,137 particles were auto-picked using the template picker and 266,357 raw particles were extracted with a box size of 512 pixels in cryoSPARC. The following 2D, 3D classifications, and refinements were all performed in cryoSPARC. 70,519 particles were selected after two rounds of 2D classification, and these particles were used to do Ab-Initio reconstruction in six classes. Then these 6 classes were used as 3D volume templates for heterogeneous refinement with all selected particles, with 52,880 particles converged into the SARS-CoV-2 S-13G9 Fab class. Next, this particle set was used to perform non-uniform refinement, yielding a resolution of $3.92 \AA$.

Local resolution estimation, filtering, and sharpening were also carried out using cryoSPARC. The full cryo-EM data processing workflow is described in Extended Data Fig. 5 and the model refinement statistics can be found in Extended Data Table 2.

Model Building and Refinement 
To build the structures of the SARS-CoV-2 S-58G6 Fab and spike-13G9 Fab complexes, the structure of the SARS-CoV-2 S glycoprotein in complex with the $\mathrm{C} 105$ neutralizing antibody Fab fragment ${ }^{28}$ (PDB: $6 \mathrm{XCN}$ ) was placed and rigid-body fitted into the cryo-EM electron density maps using UCSF Chimera ${ }^{37}$, respectively. Both of the $58 \mathrm{G} 6$ and $13 \mathrm{G} 9$ Fab models were first predicted using Phyre ${ }^{30}$ and then manually built in Coot $0.9^{38}$ with the guidance of the cryo-EM electron density maps, and overall realspace refinements were performed using Phenix $1.18^{39}$. The data validation statistics are shown in Extended Data Table 2.

\section{Creation of Figures}

Figures of molecular structures were generated using PyMOL ${ }^{40}$ and UCSF ChimeraX ${ }^{41}$.

\section{Data analysis}

Data are shown as mean \pm SEM. Two-group comparisons were performed by Student's t-test. The difference was considered significant if $p<0.05$.

\section{Declarations}

Acknowledgments This study was supported by the Emergency Project from Chongqing Medical University and Chongqing Medical University fund (X4457) with the donation from Mr Yuling Feng. We acknowledge the clinical laboratories of Yongchuan Hospital of Chongqing Medical University and the Third Affiliated Hospital of Chongqing Medical University for providing blood samples. We also thank all healthy individuals participated in this study.

Author contributions A.J. and A.H. conceived and designed the study. F.L. and H.J. were responsible for antibody production and purification. J.W., K.W., J.H., S.L., N.T., G.Z. and Q.G. conducted the pseudovirus neutralization assays and Y.X., C.G., Y.W., W.X., X.C., D.Q. and Z.Y. performed authentic SARS-CoV-2 neutralization assays. S.L. and Y.H. played an import role in data analysis of neutralizing Abs sequences. T.L., Y.W., Y.L., S.S., Q.C., F.G. and M.S. performed ELISA, competitive ELISA and peptide ELISA. X.H., C.H., R.W. and S.M. were responsible for SPR assay for the affınity of these neutralizing Abs and competition of these neutralizing Abs with ACE2. H.G., F.L., Y.G., W.W., X.J. and H.Y. carried out the cryo-EM studies. L.L. and C.H. generated figures and tables, and take responsibility for the integrity and accuracy of the data presentation. A.J., T.L. and W.W. wrote the manuscript.

Declaration of Interests: Patent has been filed for some of the antibodies presented here.

Data availability: The coordinates and structure factor files for the 13G9/SARS-CoV-2 S-RBD complex and 58G6/SARS-CoV-2 S-RBD complex are prepared for submitting in the Protein Data Bank (PDB).

\section{References}


1. Weisblum, Y., et al. Escape from neutralizing antibodies by SARS-CoV-2 spike protein variants. eLife 9(2020).

2. Korber, B., et al. Tracking Changes in SARS-CoV-2 Spike: Evidence that D614G Increases Infectivity of the COVID-19 Virus. Cell 182, 812-827.e819 (2020).

3. Wang, P., et al. Increased Resistance of SARS-CoV-2 Variants B.1.351 and B.1.1.7 to Antibody Neutralization. bioRxiv, 2021.2001.2025.428137 (2021).

4. Wibmer, C.K., et al. SARS-CoV-2 501Y.V2 escapes neutralization by South African COVID-19 donor plasma. bioRxiv, 2021.2001.2018.427166 (2021).

5. Li, S., et al. Immune characteristics analysis reveals two key inflammatory factors correlated to the expressions of SARS-CoV-2 S1-specific antibodies. Genes \& Diseases (2020).

6. Hu, J., et al. Emerging SARS-CoV-2 variants reduce neutralization sensitivity to convalescent sera and monoclonal antibodies. bioRxiv, 2021.2001.2022.427749 (2021).

7. Santos, J.C. \& Passos, G.A. The high infectivity of SARS-CoV-2 B.1.1.7 is associated with increased interaction force between Spike-ACE2 caused by the viral N501Y mutation. bioRxiv, 2020.2012.2029.424708 (2021).

8. Lauring, A.S. \& Hodcroft, E.B. Genetic Variants of SARS-CoV-2-What Do They Mean? JAMA (2021).

9. Tegally, H., et al. Emergence and rapid spread of a new severe acute respiratory syndrome-related coronavirus 2 (SARS-CoV-2) lineage with multiple spike mutations in South Africa. medRxiv, 2020.2012.2021.20248640 (2020).

10. Wang, Q., et al. Structural and Functional Basis of SARS-CoV-2 Entry by Using Human ACE2. Cell 181, 894-904.e899 (2020).

11. Walls, A.C., et al. Structure, Function, and Antigenicity of the SARS-CoV-2 Spike Glycoprotein. Cell 181, 281-292.e286 (2020).

12. Wrapp, D., et al. Cryo-EM structure of the 2019-nCoV spike in the prefusion conformation. Science $367,1260-1263$ (2020).

13. Lan, J., et al. Structure of the SARS-CoV-2 spike receptor-binding domain bound to the ACE2 receptor. Nature 581, 215-220 (2020).

14. Yi, C., et al. Key residues of the receptor binding motif in the spike protein of SARS-CoV-2 that interact with ACE2 and neutralizing antibodies. Cellular \& Molecular Immunology 17, 621-630 (2020).

15. Gavor, E., Choong, Y.K., Er, S.Y., Sivaraman, H. \& Sivaraman, J. Structural Basis of SARS-CoV-2 and SARS-CoV Antibody Interactions. Trends in immunology 41, 1006-1022 (2020).

16. Chi, X., et al. A neutralizing human antibody binds to the N-terminal domain of the Spike protein of SARS-CoV-2. Science 369, 650-655 (2020).

17. Ju, B., et al. Human neutralizing antibodies elicited by SARS-CoV-2 infection. Nature 584, 115-119 (2020).

18. Cao, Y., et al. Potent Neutralizing Antibodies against SARS-CoV-2 Identified by High-Throughput Single-Cell Sequencing of Convalescent Patients' B Cells. Cell 182, 73-84.e16 (2020). 
19. Liu, L., et al. Potent neutralizing antibodies against multiple epitopes on SARS-CoV-2 spike. Nature 584, 450-456 (2020).

20. Robbiani, D.F., et al. Convergent antibody responses to SARS-CoV-2 in convalescent individuals. Nature 584, 437-442 (2020).

21. Zost, S.J., et al. Potently neutralizing and protective human antibodies against SARS-CoV-2. Nature 584, 443-449 (2020).

22. Shi, R., et al. A human neutralizing antibody targets the receptor-binding site of SARS-CoV-2. Nature 584, 120-124 (2020).

23. Baum, A., et al. Antibody cocktail to SARS-CoV-2 spike protein prevents rapid mutational escape seen with individual antibodies. Science 369, 1014-1018 (2020).

24. Hansen, J., et al. Studies in humanized mice and convalescent humans yield a SARS-CoV-2 antibody cocktail. Science 369, 1010-1014 (2020).

25. Jiang, S., Zhang, X., Yang, Y., Hotez, P.J. \& Du, L. Neutralizing antibodies for the treatment of COVID19. Nature Biomedical Engineering 4, 1134-1139 (2020).

26. Tortorici, M.A., et al. Ultrapotent human antibodies protect against SARS-CoV-2 challenge via multiple mechanisms. Science 370, 950-957 (2020).

27. Barnes, C.O., et al. SARS-CoV-2 neutralizing antibody structures inform therapeutic strategies. Nature 588, 682-687 (2020).

28. Barnes, C.O., et al. Structures of Human Antibodies Bound to SARS-CoV-2 Spike Reveal Common Epitopes and Recurrent Features of Antibodies. Cell 182, 828-842.e816 (2020).

29. Han, X., et al. A rapid and efficient screening system for neutralizing antibodies and its application for the discovery of potent neutralizing antibodies to SARS-CoV-2 S-RBD. bioRxiv, 2020.2008.2019.253369 (2020).

30. Kelley, L.A., Mezulis, S., Yates, C.M., Wass, M.N. \& Sternberg, M.J.E. The Phyre2 web portal for protein modeling, prediction and analysis. Nature Protocols 10, 845-858 (2015).

31. Shang, J., et al. Structural basis of receptor recognition by SARS-CoV-2. Nature 581, 221-224 (2020).

32. ter Meulen, J., et al. Human monoclonal antibody combination against SARS coronavirus: synergy and coverage of escape mutants. PLoS medicine 3, e237 (2006).

33. Ou, X., et al. Characterization of spike glycoprotein of SARS-CoV-2 on virus entry and its immune cross-reactivity with SARS-CoV. Nature Communications 11, 1620 (2020).

34. Xiong, X., et al. A thermostable, closed SARS-CoV-2 spike protein trimer. Nature Structural \& Molecular Biology (2020).

35. Zheng, S.Q., et al. MotionCor2: anisotropic correction of beam-induced motion for improved cryoelectron microscopy. Nature Methods 14, 331-332 (2017).

36. Punjani, A., Rubinstein, J.L., Fleet, D.J. \& Brubaker, M.A. cryoSPARC: algorithms for rapid unsupervised cryo-EM structure determination. Nature Methods 14, 290-296 (2017). 
37. Pettersen, E.F., et al. UCSF Chimera-A visualization system for exploratory research and analysis. Journal of Computational Chemistry 25, 1605-1612 (2004).

\section{Figures}

a

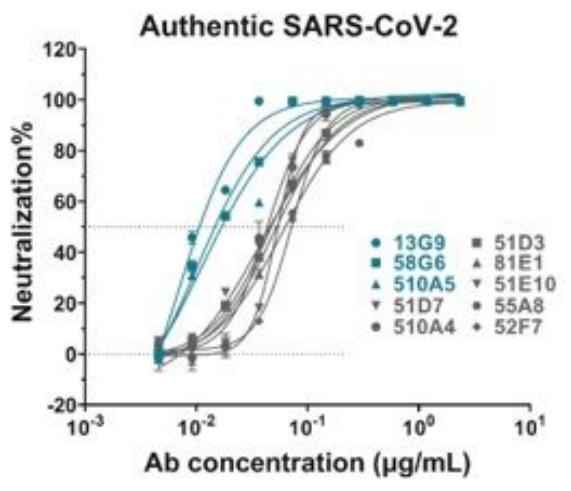

b


\section{Figure 1}

Assessment of the neutralizing capabilities of selected mAbs against SARS-CoV-2 and its variants. (a) Neutralizing potencies of the top 10 mAbs measured by authentic SARS-CoV-2 neutralization assay. (b) Neutralizing potencies of the top 10 mAbs against SARS-CoV-2, 501Y.V1 and 501Y.V2, measured by 
pseudovirus neutralization assay. Dashed lines indicated $0 \%$ and $50 \%$ reduction in the viral infectivity. Data for each $\mathrm{mAb}$ were obtained from a representative neutralization experiment, with three replicates, presented as mean \pm SEM.

a

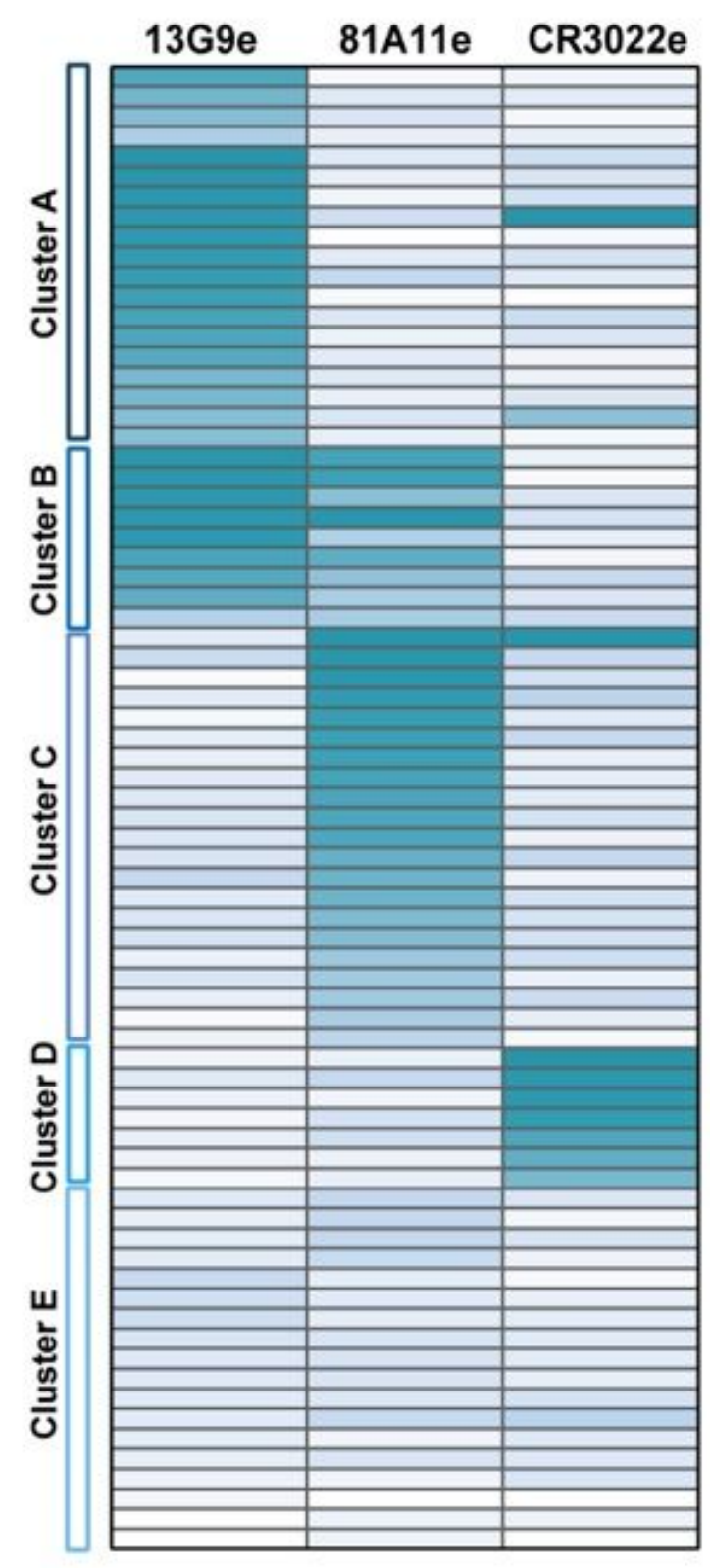

b

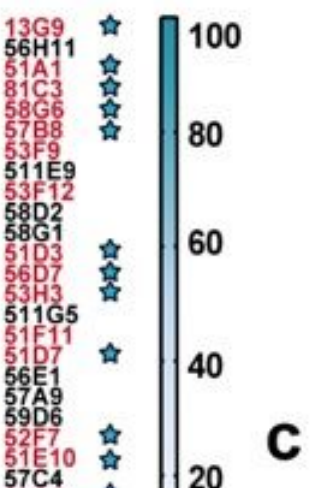

20

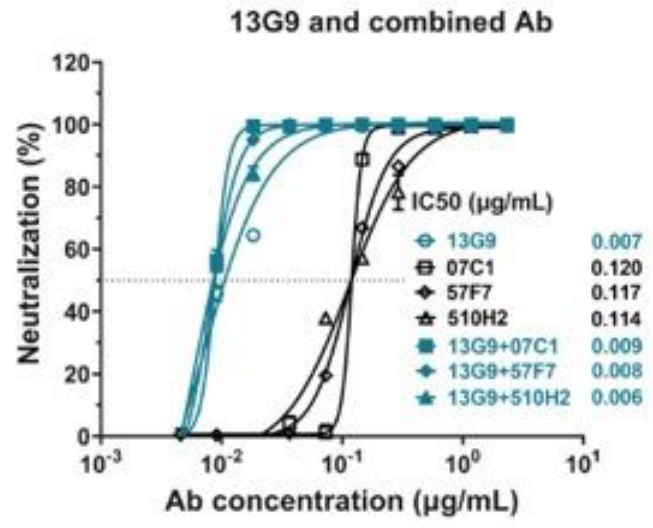

$58 \mathrm{G} 6$ and combined $\mathrm{Ab}$

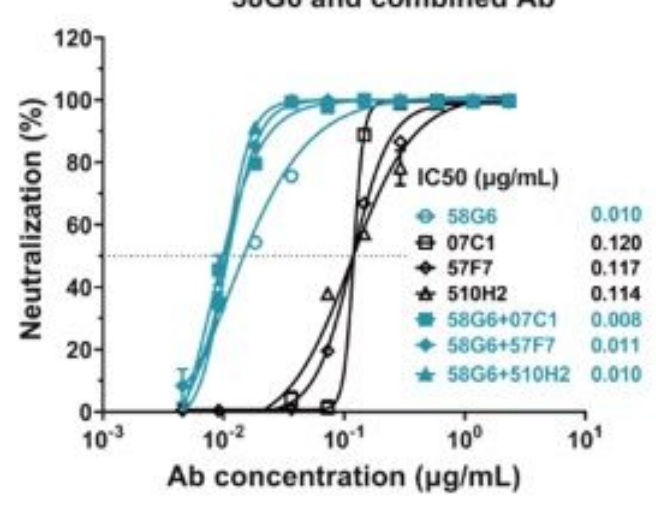

d

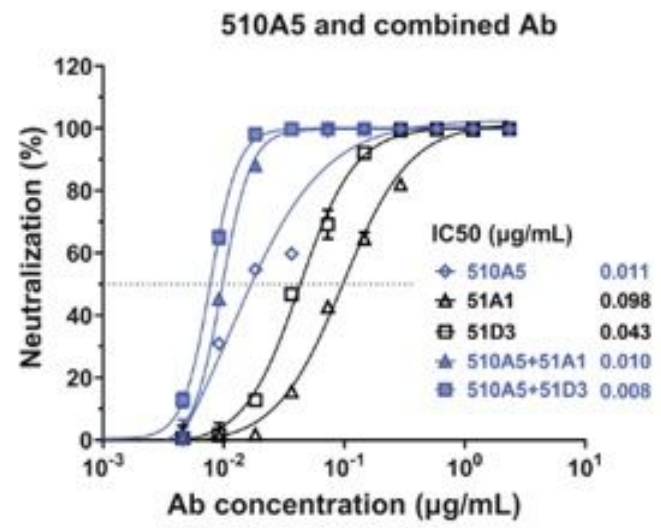

Figure 2

Epitope mapping of mAbs and the analysis of neutralizing Abs from different clusters. (a) Epitope mapping of purified mAbs targeting three independent epitopes (13G9e, 81A11e and CR3022e). All mAbs in the Cluster A competed with 13G9; each mAb in the Cluster C competed with 81A11; Cluster B consisted of mAbs cross-reacted with $13 \mathrm{G} 9 \mathrm{e}$ and $81 \mathrm{~A} 11 \mathrm{e}$, the latter to a lesser extent; all mAbs in the Cluster D targeted the epitopes overlapping with CR3022e, and the mAbs in the Cluster E recognized none 
of these 3 epitopes. All neutralizing Abs identified by authentic SARS-CoV-2 CPE assay or SARS-CoV-2 pseudovirus neutralizing assay were labelled in red. The top $20 \mathrm{mAbs}$ identified by RT-qPCR with authentic SARS-CoV-2 were indicated by blue stars. (b, c) The combination effects of 13G9 (b) and $58 \mathrm{G} 6$ (c) with $07 \mathrm{C} 1$ or $57 \mathrm{~F} 7$ recognizing $81 \mathrm{~A} 11 \mathrm{e}$, or $510 \mathrm{H} 2$ with no clearly identified epitope, against authentic SARS-CoV-2, quantified by RT-qPCR. (d) The combination effects of 510A5 with 51A1 or 51D3 recognizing $13 \mathrm{G} 9 \mathrm{e}$, against authentic SARS-CoV-2, quantified by RT-qPCR. Dashed lines indicated $50 \%$ reduction in the viral infectivity. Data for each $m A b$ were obtained from a representative neutralization experiment, with three replicates, presented as mean \pm SEM. 
a


b

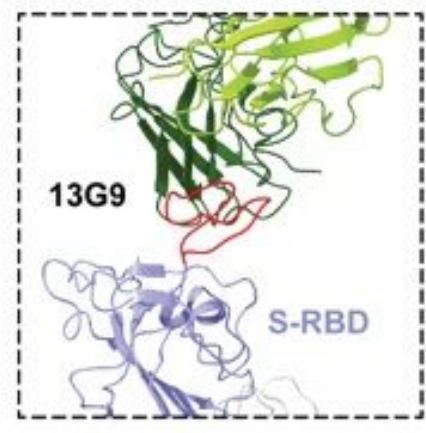

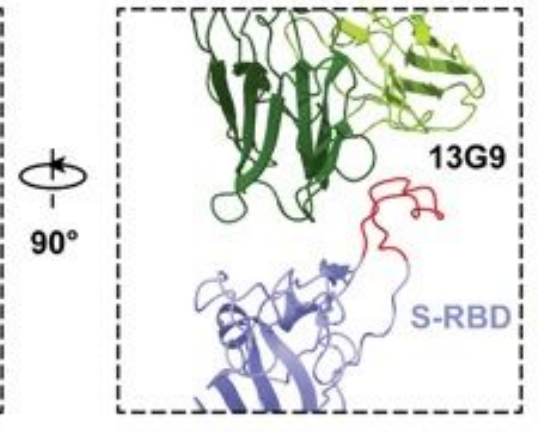

d

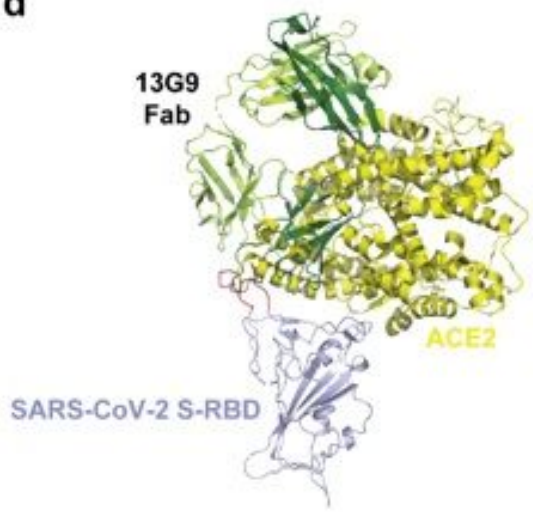

C
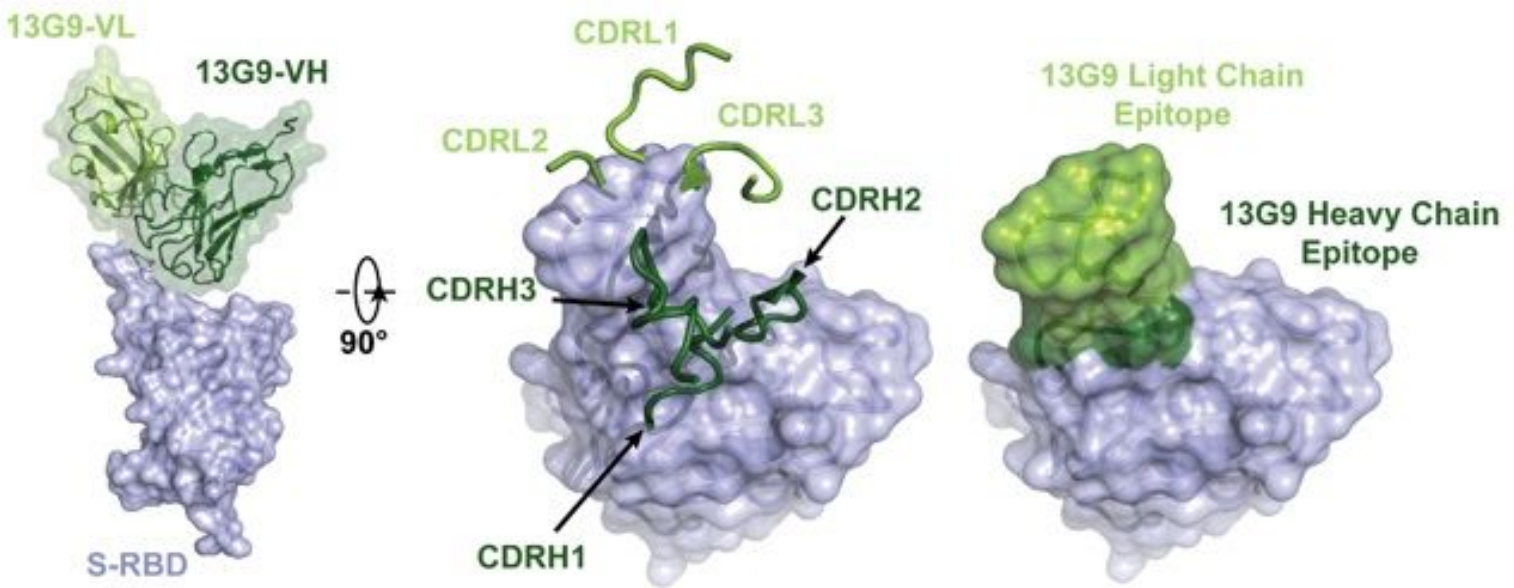

\section{Figure 3}

Cryo-EM reconstructions of the SARS-CoV-2 S trimer in complex with 13G9. (a) Orthogonal views of SARS-CoV-2 S trimer with 3 S-RBDs in the all 'up' state, complexed with 3 Fabs of 13G9. (b) Cartoon representations of the structure of SARS-CoV-2 S-RBD in complex with 13G9. The interaction loop of SRBD (S472-490) is colored in red. (c) Cartoon representation of the VH-VL domains of 13G9 bound to an S-RBD (left). CDR loops of 13G9 overlaid on surface representation of S-RBD (shown as a purple surface) 
(middle). S-RBD surface showing contacts by $13 \mathrm{G} 9 \mathrm{VH}-\mathrm{VL}$ (right). (d) Superimposition of S-RBD-13G9 Fab, together with S-RBD-ACE2 [Protein Data Bank (PDB) ID 6LZG]. All molecules are shown in cartoon representation. ACE2 is colored in yellow. All structures are presented as molecular surfaces with different colors for 3 S-RBDs (light purple), the S1 subunits (light grey) and the S2 subunits (dark grey) of the S trimers, and the 13G9 Fab light (lime) and heavy (deep green) chains.

a
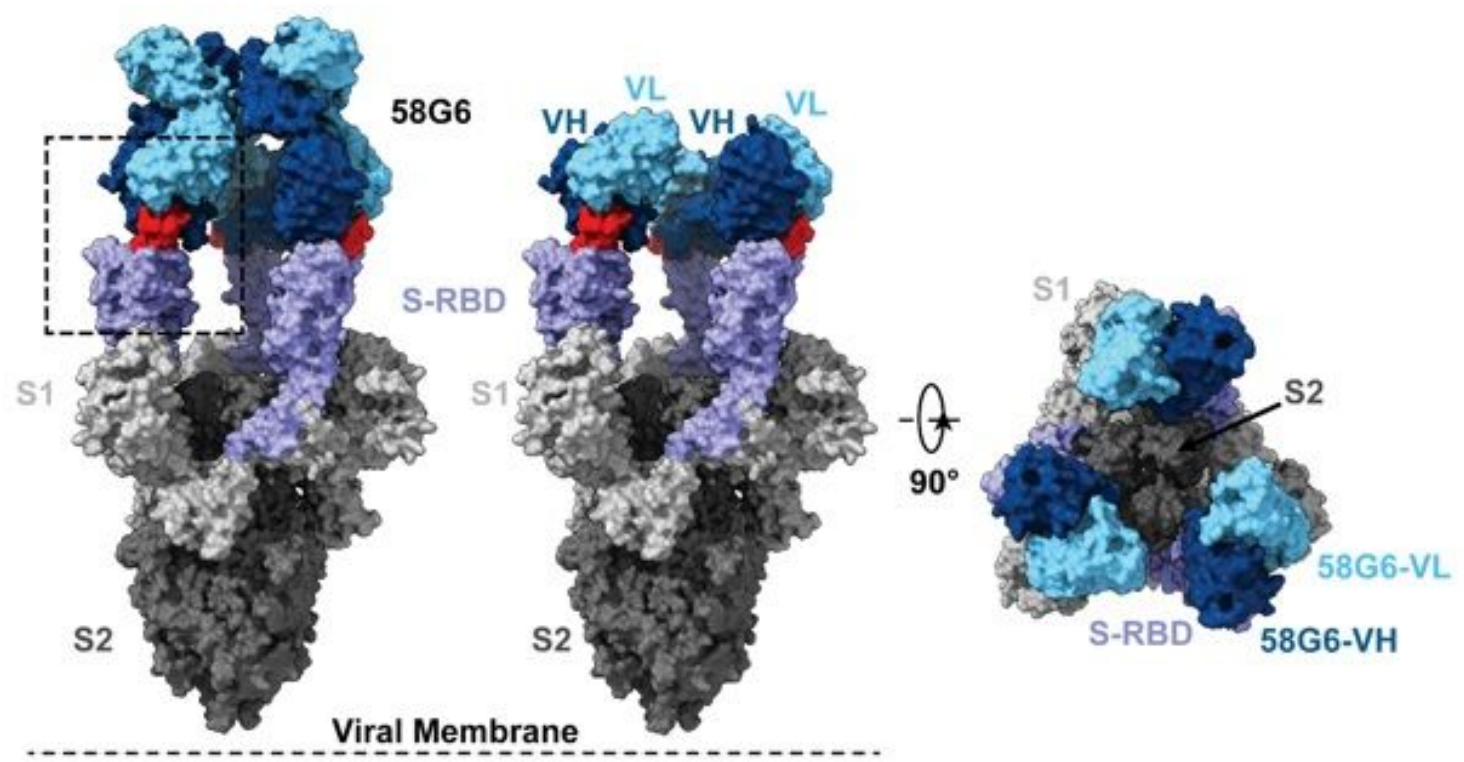

b
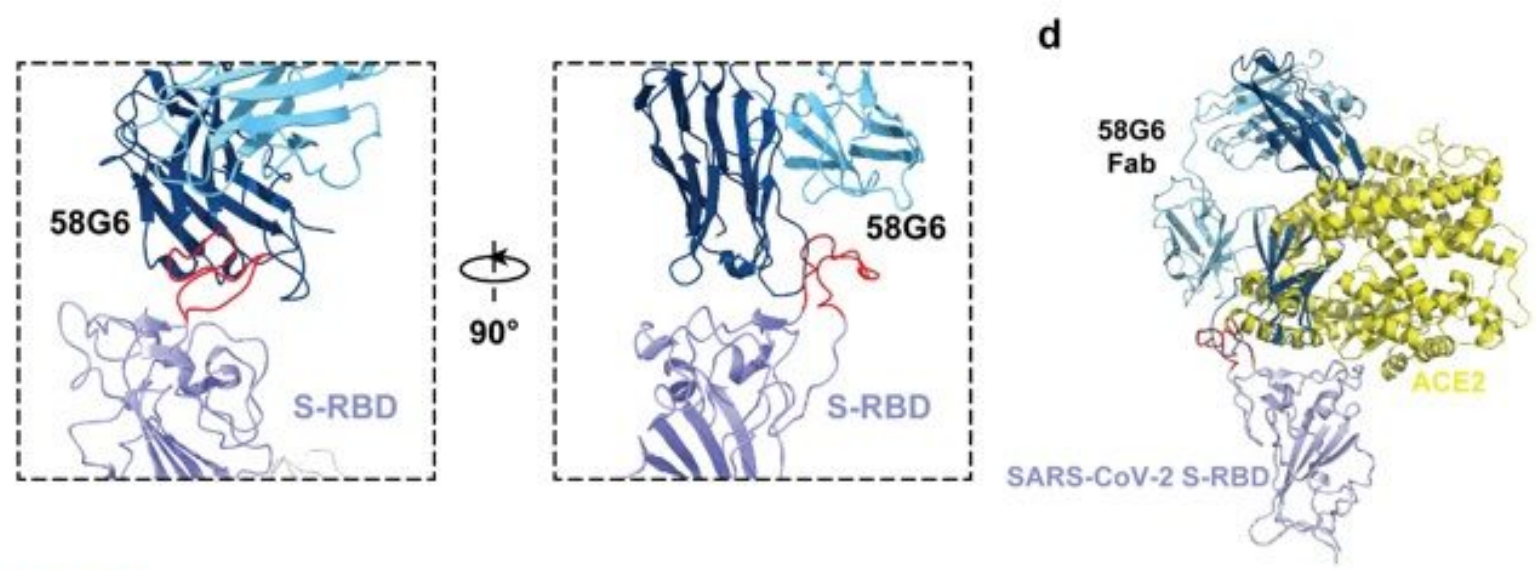

C
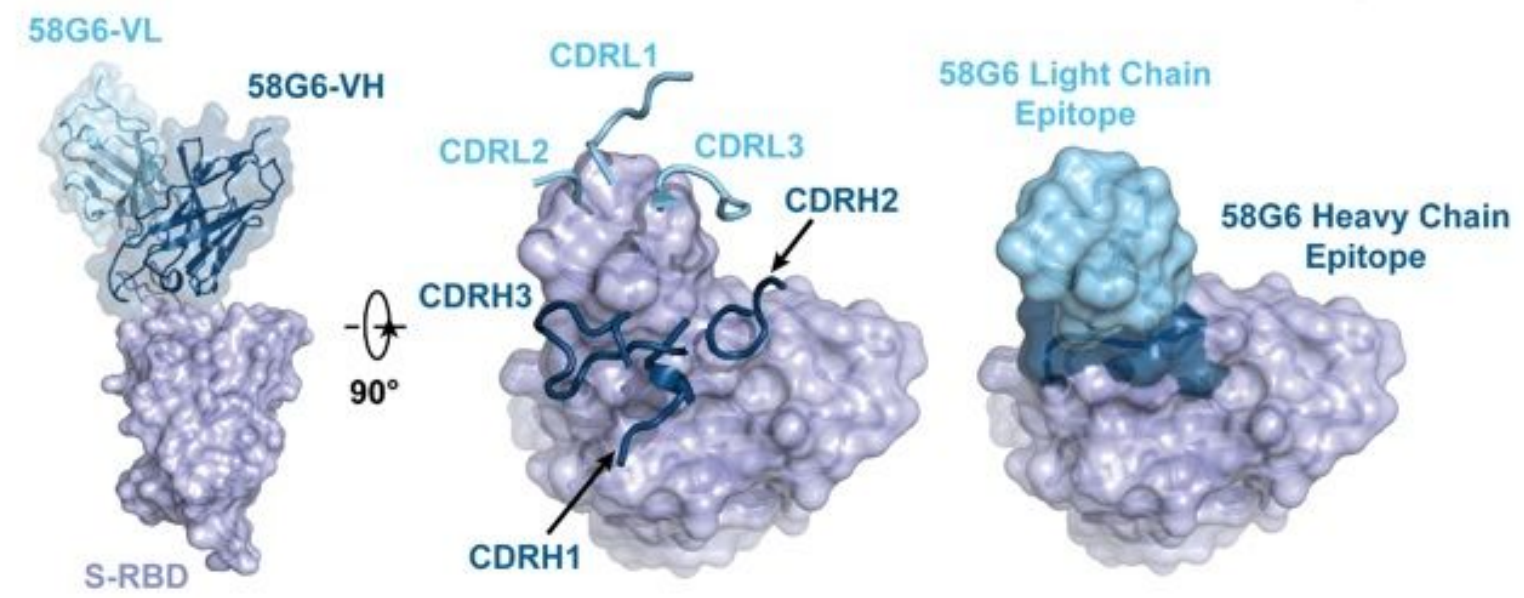

Figure 4 
Cryo-EM reconstructions of the SARS-CoV-2 S trimer in complex with 58G6. (a) Orthogonal views of SARS-CoV-2 S trimer with 3 S-RBDs in the all 'up' state, complexed with 3 Fabs of 58G6. (b) Cartoon representations of the structure of SARS-CoV-2 S-RBD in complex with 58G6. The interaction loop of SRBD (S472-490) is colored in red. (c) Cartoon representation of the VH-VL domains of $58 \mathrm{G} 6$ bound to an S-RBD (left). CDR loops of $58 \mathrm{G} 6$ overlaid on surface representation of S-RBD (shown as a purple surface) (middle). S-RBD surface showing contacts by $58 \mathrm{G} 6 \mathrm{VH}-\mathrm{VL}$ (right). (d) Superimposition of S-RBD-58G6 Fab, together with S-RBD-ACE2 [Protein Data Bank (PDB) ID 6LZG]. All molecules are shown in cartoon representation. ACE2 is colored in yellow. All structures are presented as molecular surfaces with different colors for three S-RBDs (light purple), the S1 (light grey) and S2 (dark grey) subunits of the S trimers, and the 58G6 Fab light (cyan) and heavy (deep blue) chains.

a

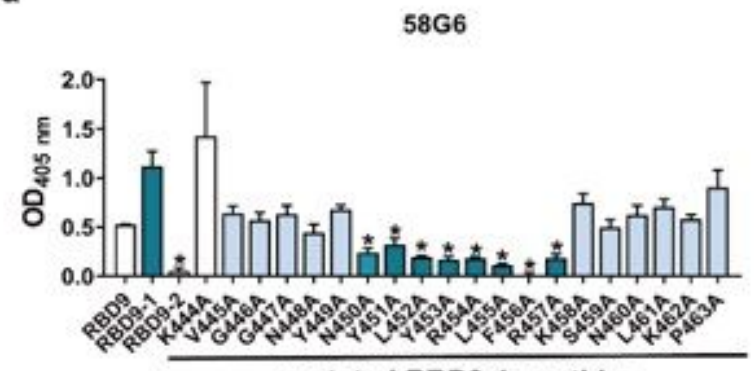

mutated RBD9-1 peptides

c

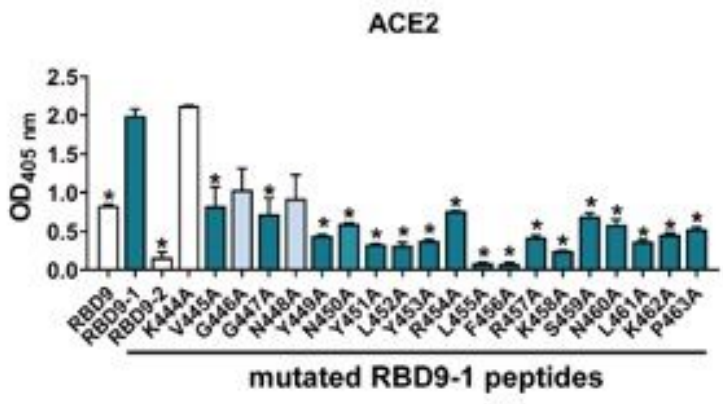

b

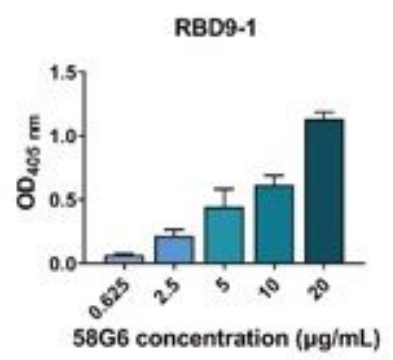

d

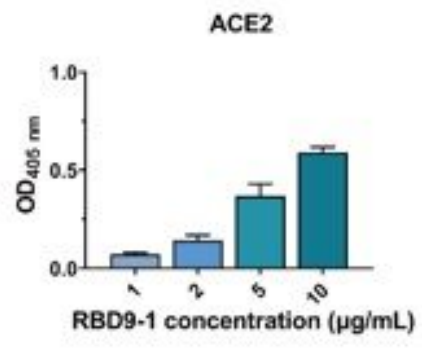

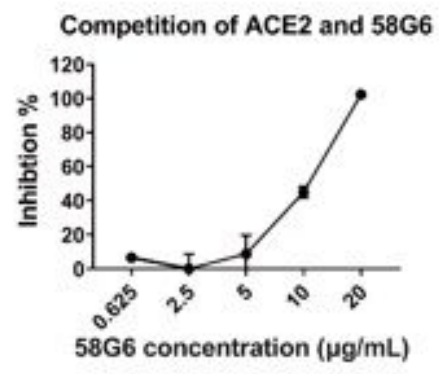

Figure 5

58G6 interacted with a linear region within the S-RBD-ACE2 binding site. ELISA result of the binding activities of 58G6 (a) or ACE2 (c) to 3 peptides covering sequences in close proximity, RBD9, RBD9-1 and RBD9-2, and single mutations derived from the full length RDB9-1. The binding activity of $58 \mathrm{G} 6$ (b) or ACE2 (d) in various concentrations to the RBD9-1 peptide, tested by ELISA. (e) The ability of 58G6 in blocking the interaction between RBD9-1 and ACE2, tested by competitive ELISA. Data are representative of at least 2 independent experiments performed in technical duplicate. The mean \pm SEM of duplicates are shown. *, $p<0.05$.

\section{Supplementary Files}

This is a list of supplementary files associated with this preprint. Click to download. 
- 20210203ExtendedDataFiguresandTables.docx

- TingtingLinreditorialpolicychecklistsignature16129361871.pdf

- flatLirs.pdf 